\title{
Catheter-directed thrombolysis versus percutaneous mechanical thrombectomy in the management of acute limb ischemia: a single center review
}

Ravi Kumar Muli Jogi1 ${ }^{1,2 *}$, Karthikeyan Damodharan ${ }^{1}$, Hing Lun leong ${ }^{1}$, Allison Chek Swee Tan', Sivanathan Chandramohan ${ }^{1}$, Nanda Kumar Karaddi Venkatanarasimha', Farah Gillan Irani ${ }^{1}$, Ankur Patel ${ }^{1}$, Apoorva Gogna', Kiang Hiong Tay ${ }^{1}$ and Thijs August Johan Urlings ${ }^{1}$

\begin{abstract}
Background: Acute limb ischemia is associated with significant mortality and amputation rate. Early restoration of flow can be obtained by various treatment methods that include catheter-directed thrombolysis (CDT) and percutaneous mechanical thrombectomy (PMT). These treatments have been shown to be effective but associated with various complications. There is lack of data comparing these two treatments. We aim to review our experience in the treatment of acute limb ischemia (ALI) and compare CDT with PMT.

Results: A total of 94 patients [mean age 65 years, 67\% male $(n=63)$ ] presented with ALI between 2006 and 2015 and were treated with either CDT or PMT. Outcomes were retrospectively reviewed. Primary outcomes were technical and clinical success; secondary outcomes were amputation rate at 30 days, duration of hospitalization and 30-day mortality. A total of 117 procedures were performed in 94 patients: 27 surgical bypass grafts, 31 previously stented arteries and 59 native vessels. Twenty eight procedures (24\%) were performed with PMT, and 89 (76\%) procedures were performed with CDT. Higher technical success was achieved in the PMT group $(68 \%, 19 / 28)$ compared to the CDT group (47\%, 42/89), $p=0.056$. Clinical success was similar in both groups $(75 \%, 21 / 28$ in the PMT group and $73 \%, 65 / 89)$ in the CDT group $(p=0.837)$. There was no statistically significant difference in 30-day mortality between the PMT vs CDT groups ( $4 \%$ vs $8 \%, p=0.425$ ). The length of post-procedural hospital stay was shorter in patients with PMT (6.0 vs 12.6 days, $p=0.001$ ). The absence of end-stage renal failure appears to be a predictor for clinical succes (HR 3.3, 95\% Cl 0.809-13.592).
\end{abstract}

Conclusion: PMT is associated with higher technical success and significantly shorter length of stay compared to CDT; however, clinical success is similar across both treatment entities. The safety profile is comparable.

Keywords: Acute limb ischemia, Thrombectomy, Thrombolysis

\footnotetext{
* Correspondence: drravikumar@live.com

${ }^{1}$ Singapore General Hospital, Hospital drive, Singapore 169608, Singapore

${ }^{2}$ Perth, Australia
} 


\section{Introduction}

Acute limb ischemia (ALI) is defined as a sudden significant decrease in limb perfusion that has been present for less than 14 days that is causing a threat to limb viability (Patel et al., 2003). To preserve viability and to reduce mortality and morbidity, early restoration of blood flow is crucial. This can be achieved by various surgical or endovascular techniques depending on the severity (Norgren et al., 2007).

Catheter directed thrombolysis (CDT) has been the first line treatment for acute limb ischaemia since the STILE and TOPAS trials in the 1990s (The STILE investigators, 1994; Ouriel et al., 1996). CDT is currently indicated in patients who do not have contraindications and who fall under I and IIA-IIB Rutherford classification categories (Byrne et al., 2014) for ALI. CDT requires long infusion times and close monitoring in an intensive care unit. Major risks of CDT include hemorrhage, including up to a $0.4 \%$ risk of potentially fatal intracranial haemorrhage when treated with Urokinase (Karnabatidis et al., 2011). Despite treatment, ALI is still associated with significant mortality (9-15\%) and an amputation rate reaching 30\% (Dormandy et al., 1999).

Due to these associated risks, percutaneous mechanical thrombectomy (PMT) devices have gained popularity. Some studies have supported the use of PMT as a first- line treatment option for ALI because this can reduce the procedure time and rapidly improve perfusion (Leung et al., 2015a).

We aim to compare the efficacy and safety profile of CDT and PMT in the management of acute limb ischemia.

\section{Materials and methods}

This is a retrospective study approved by the institutional review board in a tertiary referral hospital. The data from 117 procedures performed in 94 patients between 2006 and 2015 was collected. Treatment choice (CDT or PMT) in every case was based on the clinical scenario and the operator's expertise in PMT use.

Primary outcomes were technical and clinical success.

Technical success was defined as removal of $99 \%$ of clot.

Clinical success was defined as a return to premorbid Rutherford score without major amputation within 30 days.

The use of minor additional techniques to remove clots, such as balloon maceration and aspiration (without dedicated suction catheters), was considered part of the primary procedure in either group. Additional CDT after PMT or vice versa was deemed technical failure. The use of protective filter was not part of the treatment.

Secondary outcomes were amputation rate at 30 days, duration of hospitalization and 30-day mortality. The complications were recorded according to the CIRSE classification of complications (Filippiadis et al., 2017).

\section{Treatment}

CDT was performed according to the department protocol by infusing Urokinase (China chemicals, Hsinchu, Taiwan) via the infusion catheter in the thrombus (Craig McNamara catheter, Medtronic, USA). The typical dose of Urokinase is 2000 units/minute (120,000 units/hour). Simultaneously, heparin was infused intravenously starting at a rate of 800 units/hour. The patient returned to the angiography suite after $6 \mathrm{~h}$ for assessment and further intervention if needed.

ROTAREX (Straub Medical, Wangs, Switzerland) and ANGIOJET (Boston Scientific, Massachusetts, USA) were the two mechanical thrombectomy devices used at our institution during the study period. ANGIOJET was first used in power pulse mode using 120,000-240,000 units of Urokinase before switching to thrombectomy mode. Adjunctive procedures such as angioplasty were performed as appropriate.

\section{Statistical analysis}

Pearson's Chi-square test was used to analyze the categorical data, and a T-test was used to analyze the continuous data $(P<0.05$ was taken to be significant). Binary logistic regression was performed to identify predictors for technical and clinical success ( $p$ value $<0.2$ was taken to be significant in univariate analysis).

Multivariate logistic regression was performed, done for the significant predictors from the univariate analysis (a $P$ value $<0.05$ was taken to be significant).

\section{Results}

A total of 117 procedures were performed in 94 patients [mean age 65 years, $67 \%$ male $(n=63)$ ]. Of the 117,27 were surgical bypass grafts, 31 were previously stented arteries and 59 were native vessels. 28 procedures (24\%) utilized PMT, and 89 (76\%) procedures utilized CDT. Of the 28 procedures in the PMT group, 10 were performed with ROTAREX and 18 were performed with ANGIOJET.

Both groups were comparable in terms of age, gender and co-morbidities (see Table 1).

The characteristics of clinical presentation and location of the occlusion are presented in Table 2. The duration of symptoms was comparable between both groups; however, there was a tendency for a lower Rutherford classification for ALI in the PMT group [category I and IIA in $82 \%(23 / 28)$ ] than the CDT group $[63 \%(56 / 89)]$ with a $p$ value of 0.058 . More native vessels $[59.6 \%(53 / 89)$ vs $29 \%(8 / 28)]$ and bypasses $[27 \%$ $(24 / 89)$ vs $14 \%(4 / 28)]$ were treated with CDT. By chance, the majority of stented vessels were in PMT group [57\% (16/28) vs $13.4 \%(12 / 89)]$. In the CDT group, $57 \%(50 / 89)$ of patients were treated for femorocrural occlusions compared to $25 \%(7 / 28)$ in the PMT group. 
Table 1 Population characteristics

\begin{tabular}{llll}
\hline & PMT $(n=28)$ & CDT $(n=89)$ & p value \\
\hline Mean age +/- SD & $63.3+/-10.5$ & $66.0+/-12.4$ & 0.310 \\
$\quad$ Male gender & $18(64.3 \%)$ & $60(67.4 \%)$ & 0.759 \\
$\begin{array}{l}\text { AGE GROUP } \\
\text { 30-50 years }\end{array}$ & $3(10.7 \%)$ & $12(13.5 \%)$ & 0.382 \\
51-70 years & $18(64.3 \%)$ & $44(49.4 \%)$ & \\
71-90 years & $7(25 \%)$ & $33(37.1 \%)$ & \\
CO-MORBIDITIES & & & \\
Diabetes mellitus & $16(57.1 \%)$ & $55(61.8 \%)$ & 0.660 \\
Hypertension & $21(75 \%)$ & $67(75.3 \%)$ & 0.976 \\
IHD & $11(39.3 \%)$ & $51(57.3 \%)$ & 0.096 \\
CVA & $4(14.3 \%)$ & $16(8 \%)$ & 0.651 \\
Smoking & $9(32.1 \%)$ & $25(28.1 \%)$ & 0.680 \\
ESRF & $3(10.7 \%)$ & $6(6.7 \%)$ & 0.491 \\
\hline
\end{tabular}

In the PMT group, $71 \%(20 / 28)$ received adjunctive angioplasty compared to $64 \%(57 / 89)$ in the CDT group. The average length of occlusion in PMT group was 25 $\mathrm{cm}$ and $34.5 \mathrm{~cm}$ in CDT group. The mean duration of CDT was $11.9 \mathrm{~h}$ with a standard deviation of $8.48 .36 \%$ patients (10/28) in PMT group and 31\% (28/89) in CDT group had antegrade puncture.

\section{Primary and secondary outcomes}

Primary and secondary outcomes are presented in Table 3. Higher technical success was achieved in the PMT group $(68 \%, 19 / 28)$ compared to the CDT group

\begin{tabular}{|c|c|c|c|}
\hline & PMT $(n=28)$ & CDT $(n=89)$ & $p$ value \\
\hline \multicolumn{4}{|l|}{ DURATION OF SYMPTOMS } \\
\hline Less than $24 \mathrm{~h}$ & $5(17.9 \%)$ & $13(14.6 \%)$ & 0.338 \\
\hline 1 to 7 days & $12(42.9 \%)$ & $52(58.4 \%)$ & \\
\hline 8 to 14 days & $11(39.25)$ & $24(27 \%)$ & \\
\hline \multicolumn{4}{|l|}{ RUTHERFORD CLASSIFICATION } \\
\hline I and IIA & $23(82.1 \%)$ & $56(62.9 \%)$ & 0.058 \\
\hline$\| \mathrm{B}$ & $5(17.9 \%)$ & $33(37.1 \%)$ & \\
\hline \multicolumn{4}{|l|}{ OCCLUSION } \\
\hline Thrombosed at stent & $16(57.1 \%)$ & $12(13.4 \%)$ & \\
\hline Thrombosed at bypass & $4(14.3 \%)$ & $24(27 \%)$ & \\
\hline Thrombosed at native vessel & $8(28.6 \%)$ & $53(59.6 \%)$ & \\
\hline \multicolumn{4}{|l|}{ OCCLUDED } \\
\hline \|liac & $1(3.6 \%)$ & $0(0 \%)$ & \\
\hline Iliofemoral & $1(3.6 \%)$ & $5(5.6 \%)$ & \\
\hline Femoral, popliteal & $15(53.6 \%)$ & $30(33.7 \%)$ & \\
\hline Femorocrural & $7(25 \%)$ & $50(57.3 \%)$ & \\
\hline Crural \& pedal & $4(14.3 \%)$ & $3(3.4 \%)$ & \\
\hline
\end{tabular}

Table 3 Results summary

\begin{tabular}{llll}
\hline & PMT $(n=28)$ & CDT $(n=89)$ & $p$ value \\
\hline PRIMARY OUTCOME & & & \\
Clinical success & $21(75 \%)$ & $65(73 \%)$ & 0.837 \\
Technical success & $19(67.9 \%)$ & $42(47.2 \%)$ & 0.056 \\
SECONDARY OUTCOME & & & \\
$\quad$ Major amputation* (30 days) & $2(7.1 \%)$ & $15(16.9 \%)$ & 0.323 \\
$\quad$ Minor amputation(30 days) & $1(3.6 \%)$ & $1(1.1 \%)$ & \\
Hospitalization (mean days) & 6.0 & 12.6 & 0.001 \\
COMPLICATIONS (CIRSE) (Filippiadis et al., 2017) & & \\
$\quad$ Grade 2 & $6(21.4 \%)$ & $21(23.57 \%)$ & \\
Grade 3 & $1(3.6 \%)$ & $4(4.5 \%)$ & \\
Grade 6 & $1(3.6 \%)$ & $2(2.3 \%)$ & \\
30 Day mortality & $1(3.6 \%)$ & $7(8 \%)$ & 0.425 \\
\hline
\end{tabular}

$(47 \%, 42 / 89)(p=0.056)$. However, clinical success was similar between the groups $(75 \%, 21 / 28$ in the PMT group and $73 \%, 65 / 8$ in the CDT group $(p=0.837)$. PMT was converted to CDT in $14 \%(4 / 28)$ and CDT was converted to PMT in 6\% (5/89), which was considered treatment failure.

The major amputation at 30 days was $7.1 \%(2 / 28)$ in the PMT group vs $16.9 \%(15 / 89)$ in the CDT group $(p=0.323)$. The minor amputation rate at 30 days was $3.6 \%(1 / 28)$ in the PMT group vs $1 \%(1 / 89)$ in CDT group. The length of post procedural hospital stay was shorter in patients undergoing PMT (6.0 vs 12.6 days, $p=0.001$ ).

\section{Regression analysis for primary outcomes}

A logistic univariate and multivariate regression analysis was performed to identify significant predictors of technical and clinical success. PMT was a significant predictor for a higher technical success (HR 3.2, 95\% CI 1.0849.326, $p=0.035)$. However, the use of PMT was not found to be a significant predictor for clinical success. The absence of end-stage renal failure appears to be a predictor for clinical succes (HR 3.3, 95\% CI 0.809-13.592); however, this did not reach statistical significance $(p=0.096)$. End stage renal disease is defined by Kidney Disease Outcome Quality Initiative, as the patient being dialysis dependent (KDOQI guideline).

\section{Complications}

Complications are presented in Table 3. In the PMT group, closure device was used in $48 \%(14 / 29)$. Groin haematoma was seen in 2 patients treated with closure device. 1 patient without closure device had pseudoaneurysm and 1 patient died secondary to a massive groin haematoma. 1 patient had chest wall hematoma requiring transfusion and 1 patient had fasciotomy for compartment syndrome. Distal embolization was seen in 1 patient. 
In the CDT group, closure device was used in $45 \%$ $(40 / 89)$. Groin haematoma was seen in 7 patients with closure device and 7 patients without closure device. Gastro-intestinal bleed requiring transfusion were seen in a total of 7 patients. Compartment syndrome requiring fasciotomy was recorded in 4 patients. There were no cases of intracranial haemorrhage in the CDT group. Two deaths were recorded in this group; one secondary to acute renal failure and adult respiratory syndrome, the other as a complication of compartment syndrome. Distal embolization was seen in 2 patients.

\section{Discussion}

Our study shows higher technical success in the PMT group compared to the CDT group (68\% vs $47 \%$ ). However, CDT in this cohort was used in more complex distal occlusive disease. This is likely because the use of PMT below the knee vessels is thought to be risky due to small calibre vessels in our population. Conversion of one treatment to another was only seen in $14 \%$ of patients in PMT group and 6\% of patients in CDT group, hence, did not contribute much towards drop in technical success. The choice of treatment is also dependent on the operator's experience and knowledge of thrombectomy devices. This selection bias is inherent to the retrospective nature of this study.

Few studies have demonstrated high technical success with PMT ranging between 56 and 95\% (Karnabatidis et al., 2011). Kasirajan et al. investigated PMT using ANGIOJET in acute and subacute limb ischemia and divided technical success into failure $(<50 \%$ luminal restoration), partial success (50-95\% luminal restoration) and complete success (>95\% luminal restoration). The complete success rate was $61.4 \%$ (Kasirajan et al., 2001).

Similarly, Byrne et al. demonstrated 90\% technical success rate but they defined technical success as the restoration of inline flow to the foot without requiring immediate surgical intervention (Byrne et al., 2014), which is similar to our definition. However, we also attempted to quantify the clot burden and defined technical success as the removal of $99 \%$ of the clot.

Despite PMT being technically superior, the clinical success remains similar in the PMT and CDT groups (75\% vs $73 \%$ ) and PMT was also not a predictor for clinical success in the regression analysis.

The regression analysis showed that patients without end-stage renal disease had a tendency for higher clinical success. Patients with end-stage renal disease tend to have extensive vascular disease and revascularization can be challenging in such cases. Similar findings were reported by Byrne et al.; they reported a 6 to 26 -fold increased risk of amputation and the loss of primary and secondary patency in patients with end-stage renal disease (Byrne et al., 2014).
As previously noted, the major limiting factor for CDT is hemorrhagic complications. Seven percent $(n=6)$ of patients receiving CDT in this study had major bleeding. Of these, 3 patients had groin access hematomas and 2 patients had gastro-intestinal bleeding. Seven percent $(n=2)$ of patients receiving PMT had major groin access hematomas; this difference was not statistically significant. None of the patients receiving CDT had a hemorrhagic stroke in our cohort. Karnabatidis et al. reported a 0.4\% risk of potentially fatal intracranial hemorrhage when treated with Urokinase (Karnabatidis et al., 2011).

In this study, there was no significant difference in 30-day mortality between CDT and PMT ( $8 \%$ vs $4 \%$, $p=0.425$ ); this is comparable to the published data. Byrne et al. reported 30-day mortality in $4.8 \%$ of patients with CDT and 5.6\% with PMT ( $p=0.82)$ (Byrne et al., 2014).

The duration of hospitalization was significantly shorter in patients who underwent PMT; the mean stay was 6 days in PMT group vs 12.6 days in the CDT group, $p=0.0001$. PMT was used more in category I and IIa patients with femoral and popliteal disease. The reduced technical success in the CDT group may have played a role in prolonging the hospital stay in this group. The mean duration of CDT was $12 \mathrm{~h}$ in this study; hence, this likely did not play a major role in the duration of hospitalization.

The thirty-day amputation rates were not signficantly different between the PMT vs CDT groups (7\% vs $17 \%$, $p=0.323)$. Limb salvage rate for PMT in this study was $93 \%$, which was comparable to literature. Ansel et al. reported limb salvage rate of $89 \%$ at 1 month following PMT (Ansel et al., 2008).

This study is limited by its retrospective nature and unequal patient numbers between the PMT group and CDT group. However, there is a lack of randomized trials comparing these two treatments and the indications for these treatments in acute limb ischemia are not clear. The difference in costs between CDT and PMT were not evaluated, which may play a role in decision making because the clinical success rates are similar.

\section{Conclusion}

PMT is safe and effective as a primary treatment for ALI with a similar clinical outcome compared to CDT. Several treatment options are available for this patient group and the choice of treatment should still be on a case-by-case basis. Patients requiring quick revascularization (for example Rutherford IIB) and with contraindications for thrombolysis may benefit from PMT. Similarly, CDT can be the treatment of choice in patients with complex crural disease. A combination of these techniques should be considered when appropriate. 


\section{Abbreviations}

ALI: Acute limb ischemia; CDT: Catheter directed thrombolysis;

CIRSE: Cardiovascular and Interventional Radiological Society of Europe:

PMT: Percutaneous mechanical thrombectomy

\section{Acknowledgements}

We thank Win Hliang Hliang for her help with statistical analysis.

\section{Funding}

not applicable.

\section{Availability of data and materials}

All data generated or analysed during this study are included in this article.

\section{Author's contributions}

RMJ is the author of this article and also involved in data interpretation. TJU designed the study and interpreted data. HLL \& ACS collected data. KDA, CMS, NKV, FGI, AKP, APG and TKH proof read the article and also helped with data collection and interpretation. All authors have read and approved the final manuscript.

\section{Ethics approval and consent to participate}

This study was approved by the Institutional Review board. The need for consent was waived.

\section{Consent for publication}

not applicable.

\section{Competing interests}

The authors declare that they have no competing interests.

\section{Publisher's Note}

Springer Nature remains neutral with regard to jurisdictional claims in published maps and institutional affiliations.

Received: 15 August 2018 Accepted: 13 November 2018

Published online: 03 December 2018

\section{References}

Ansel GM, Botti CF, Silver MJ (2008) Treatment of acute limb ischaemia with a percutaneous mechanical thrombectomy-based endovascular approach: 5 year limb salvage and survival results from a single center series. Catheter Cardiovasc Interv 72(3):325-330

Byrne RM, Taha AG, Avgerinos E et al (2014) Contemporary outcomes of endovascular interventions for acute limb ischemia. J Vasc Surg 59(4):988-995

Dormandy J, Heeck L, Vig S (1999) Acute limb ischaemia. Semin Vas Surg 12:148-153

Filippiadis DK, Binkert C, Pellerin O, Hoffmann RT, Krajina A, Pereira PL (2017 Aug) Cirse quality assurance document and standards for classification of complications: the Cirse classification system. Cardiovasc Intervent Radiol 40(8):1141-1146

Karnabatidis D, Spiliopoulos S, Tsetis D et al (2011) Quality improvement guidelines for percutaneous catheter directed intra-arterial thrombolysis and mechanical thrombectomy for acute limb ischaemia. Cardiovasc Intervent Radiol 34:1123-1136

Kasirajan K, Gray B, Beavers FP et al (2001) Rheolytic thrombectomy in the management of acute and subacute limb-threatening ischemia. J Vasc Intervel Radiol 12(4):413-421

Leung DA, Blitz LR, Nelson T et al (2015a) Rheolytic pharmaco mechanical thrombectomy for the management of acute limb ischaemia: results from the PEARL registry. J Endovasc Ther 22(4):546-557

Norgren L, Hiatt WR, Dormandy JA et al (2007) Inter society consensus for the management of peripheral arterial disease (TASC II). Eur J Vasc Endovac Surg 33(1):S1-S75

Ouriel K, Veith FJ, Sasahara AA (1996) for the TOPAS investigators. Thrombolysis or peripheral arterial surgery (TOPAS): phase 1 results. J Vasc Surg 23:64-75

Patel N, Sacks D, Patel Rl et al (2003) SIR reporting standards for the treatment of acute limb ischemia with use of transluminal removal of arterial thrombus. J Vasc Interv Radiol 14:S453-S465

The STILE investigators (1994) Results of a prospective randomised trial evaluating surgery versus thrombolysis for ischaemia of the lower extremity. The STILE trial. Ann Surg 220:251-268

\section{Submit your manuscript to a SpringerOpen ${ }^{\circ}$ journal and benefit from:}

- Convenient online submission

- Rigorous peer review

- Open access: articles freely available online

- High visibility within the field

- Retaining the copyright to your article

Submit your next manuscript at $\boldsymbol{\nabla}$ springeropen.com 